\title{
Impact of Histological Type and Grade on the Diagnostic Accuracy of Intraoperative Frozen Section for Detecting Breast Cancer Metastasis to Axillary Sentinel Lymph Nodes
}

\author{
Atif A. Hashmi ${ }^{1}$, Rubina Riaz ${ }^{2}$, Shamail Zia ${ }^{3}$, Hiba Shahid ${ }^{4}$, Umair Arshad Malik ${ }^{5}$, Rabeet Khan ${ }^{6}$, \\ Muhammad Irfan ${ }^{7}$, Farozaan Shamail ${ }^{3}$, Fazail Zia ${ }^{8}$, Muhammad Ghani Asif ${ }^{9}$ \\ 1. Pathology, Liaquat National Hospital and Medical College, Karachi, PAK 2. Pathology, Fazaia Medical College, Air \\ University, Islamabad, PAK 3. Pathology, Ziauddin University, Karachi, PAK 4. Internal Medicine, Liaquat National \\ Hospital and Medical College, Karachi, PAK 5. Internal Medicine, Aga Khan University, Karachi, PAK 6. Internal \\ Medicine, Buckinghamshire Healthcare NHS Trust, Aylesbury, GBR 7. Statistics, Liaquat National Hospital and Medical \\ College, Karachi, PAK 8. Pathology, Jinnah Sindh Medical University, Karachi, PAK 9. Pathology, Multan Medical and \\ Dental College, Multan, PAK
}

Corresponding author: Atif A. Hashmi, atifhashmi345@gmail.com

\section{Abstract \\ Introduction}

Intraoperative sentinel lymph node (SLN) evaluation is the standard of care in patients with clinically nodenegative breast cancer. The most common histological subtype of breast carcinoma is invasive ductal carcinoma (IDC), followed by invasive lobular carcinoma (ILC). Alternatively, histological grades vary from grades G1 to G3. Therefore, in this study, we evaluated the diagnostic accuracy of frozen section (FS) for detecting breast cancer metastasis to SLNs with respect to histological subtypes and grades.

\section{Methods}

A retrospective observational study was conducted in the Department of Histopathology at Liaquat National Hospital and Medical College, Pakistan, from January 2013 till December 2020, over a duration of eight years. A total of 540 cases of primary breast cancer, undergoing upfront breast surgery were included in the study. Intraoperatively, SLNs were identified and sent for FS. After FS reporting, the remaining tissue was submitted for final (paraffin) section examination after formalin fixation, and results of FS and final (paraffin) sections were compared.

\section{Results}

The mean age of the patients included in the study was $52.05 \pm 12.42$ years, and the median number of SLNs was three (ranging from one to 14). The overall sensitivity, specificity, positive predictive value, negative predictive value, and diagnostic accuracy of intraoperative FS were $88.2 \%, 100 \%, 100 \%, 92.5 \%$, and $95.2 \%$, respectively. The sensitivity of FS for IDC was $88.3 \%$, whereas it was $85.7 \%$ for ILC. Alternatively, the sensitivity of FS for grade G1, G2, and G3 tumors was $78.3 \%$, 91.5\%, and $90.2 \%$, respectively. The falsenegative rate for grade $\mathrm{G} 1$ tumors was $21.7 \%$, which was higher than G2 and G3 tumors ( $8.5 \%$ and $9.8 \%$, respectively). Similarly, the false-negative rate for cases where the number of SLNs was more than three was only 5.4\%, which was lower than cases with a single and two to three SLNs sent on FS (23.1 and 14.7\%, respectively).

\section{Conclusion}

The sensitivity of intraoperative FS for detecting ILC metastasis to axillary SLNs was not substantially different from IDC; however, histological grade affects the sensitivity of FS diagnosis, with lower-grade tumors having low sensitivity. Moreover, increasing the number of SLNs sent intraoperatively on FS improves the sensitivity of FS for detecting breast cancer metastasis to axillary SLNs.

Categories: Pathology, General Surgery, Oncology

Keywords: intraoperative frozen section, sentinel lymph node (sln), breast carcinoma, infiltrating ductal carcinoma, infiltrating lobular carcinoma

\section{Introduction}

Breast cancer is one of the most common cancers among women in Southeast Asia, with an increasing number of cases at a young age [1]. Breast cancer is a heterogeneous disease with many histological and molecularly distinct subtypes. Apart from the common subtypes, many rare special subtypes of breast carcinoma were defined [2-3]. Alternatively, these different subtypes vary with respect to hormone receptor 
and human epidermal growth factor receptor 2 (HER2/neu) expressions [4-5]. Apart from cancer-related morbidity, chemotherapy for breast cancer is also associated with a significant number of complications [67]. Surgical management of breast cancer has evolved over a period of years, with respect to the axillary lymph node dissection. The most important prognostic factor in breast cancer is axillary lymph node metastasis. Alternatively, axillary lymph node dissection leads to increased morbidity in cancer patients. To overcome this problem, intraoperative sentinel lymph node (SLN) evaluation has become a standard of care in patients with clinically node-negative breast cancer [8]. Negative SLN on frozen section (FS) spares a patient from unnecessary axillary lymph node dissection.

Previous studies have confirmed a good diagnostic accuracy of FS for detecting axillary lymph node metastasis [9]. However, breast cancer is a heterogeneous disease with different histological subtypes and grades [10]. It is unclear if grade and histological type affect the diagnostic accuracy of FS for detecting metastatic carcinoma in SLNs. The most common histological subtype of breast carcinoma is invasive ductal carcinoma (IDC), followed by invasive lobular carcinoma (ILC). Alternatively, histological grades vary from grade G1 to G3. Therefore, in this study, we evaluated the diagnostic accuracy of FS for detecting breast cancer metastasis in SLNs with respect to histological subtypes and grades.

\section{Materials And Methods}

A retrospective observational study was conducted in the Department of Histopathology at Liaquat National Hospital and Medical College, Pakistan, from January 2013 till December 2020, over a duration of eight years. A total of 540 cases of primary breast cancer undergoing upfront breast surgery were included in the study. All these cases had clinically negative axillary lymph nodes and biopsy-proven breast carcinoma. Cases with a histological diagnosis of either IDC or ILC of any grade were included in the study. Cases with neoadjuvant chemotherapy or radiation were excluded from the study. Immunohistochemical (IHC) staining for E-cadherin was performed on trucut biopsy to confirm the diagnosis of ILC. Intraoperatively, SLNs were identified and sent for FS. The number and size of SLNs were recorded, and SLNs were sectioned at a $2 \mathrm{~mm}$ interval perpendicular to the long axis of the lymph nodes and submitted for FS. Three step levels were examined on FS after hematoxylin and eosin ( $\mathrm{H} \& \mathrm{E})$ staining. The remaining tissue was submitted for final (paraffin) section examination after formalin fixation. One $\mathrm{H} \& \mathrm{E}$-stained section was examined on the final (paraffin) section and IHC staining was performed where necessary using cytokeratin (CKAE1/AE3) stain. The diagnosis of FS was compared with the final (paraffin) section for analysis.

Based on the diagnosis of FS, a decision regarding axillary dissection was made. For patients undergoing mastectomy, any positive SLN with macrometastasis (>2 $\mathrm{mm}$ metastatic deposit) was followed by axillary dissection. For cases undergoing breast conservation surgery, three or more positive SLNs with macrometastasis were followed by axillary dissection. In the remaining cases, axillary dissection was not performed.

After SLN resection, definitive breast surgery (lumpectomy or modified radical mastectomy) was performed, and specimens were sent to histopathology laboratory in formalin. After overnight formalin fixation, gross examination of the specimen was done and tumor size was measured. The representative sections were submitted from the tumor, resection margins, normal breast tissue, and axillary lymph nodes [11-14].

Data analysis was performed using the Statistical Package for the Social Sciences (Version 26.0, IBM Corp, Armonk, NY). Sensitivity, specificity, positive predictive value (PPV), negative predictive value (NPV), and diagnostic accuracy were calculated for FS diagnosis by $2 \times 2$ tables using the final (paraffin) section diagnosis as the gold standard.

\section{Results}

The mean age of the patients included in the study was $52.05 \pm 12.42$ years, and the median number of SLNs was three (ranging from one to 14). The most common tumor grade was G3 (41.9\%). A total of $40.7 \%$ SLNs were positive for metastatic carcinoma on final (paraffin) histology (Table 1). 


\section{Cureus}

Clinicopathological features

Age (years), mean $\pm S D$

Age groups

$\leq 50$ years, $n(\%)$

$>50$ years, $n(\%)$

Number of SLNs, median (range)

Histological type

IDC, n (\%)

ILC, n (\%)

Grade

Grade 1, n (\%)

Grade 2, n (\%)

Grade 3, n (\%)

Tumor size

$\leq 2 \mathrm{~cm}, \mathrm{n}(\%)$

$2.1-5 \mathrm{~cm}, \mathrm{n}(\%)$

$>5 \mathrm{~cm}, \mathrm{n}(\%)$

Type of surgery

Lumpectomy, n (\%)

Modified radical mastectomy, n (\%)

Simple mastectomy, n (\%)

Laterality

Right, n (\%)

Left, n (\%)

Frozen section diagnosis

Positive for metastatic carcinoma, n (\%)

Negative for metastatic carcinoma, n (\%)

Final (paraffin) section diagnosis

Positive for metastatic carcinoma, n (\%)

Negative for metastatic carcinoma, n (\%)
Values

$52.05 \pm 12.42$

288 (53.3)

252 (46.7)

3 (1-14)

468 (86.7)

72 (13.3)

100 (18.5)

214 (39.6)

226 (41.9)

68 (12.6)

416 (77)

56 (10.4)

220 (40.7)

198 (36.7)

122 (22.6)

332 (61.5)

208 (38.5)

194 (35.6)

346 (64.4)

$220(40.7)$

320 (59.3)

TABLE 1: Clinicopathological features of the population under study

SD, standard deviation; IDC, infiltrating ductal carcinoma; ILC, infiltrating lobular carcinoma

The overall sensitivity, specificity, positive predictive value (PPV), negative predictive value (NPV), and diagnostic accuracy of intraoperative FS were $88.2 \%, 100 \%, 100 \%, 92.5 \%$, and $95.2 \%$, respectively (Table 2). 


\section{Cureus}

\begin{tabular}{|c|c|c|c|c|c|c|c|c|}
\hline \multirow{2}{*}{ Frozen section diagnosis } & \multicolumn{3}{|c|}{ Final (paraffin) section diagnosis } & \multirow{2}{*}{ Sensitivity } & \multirow{2}{*}{ Specificity } & \multirow{2}{*}{ PPV } & \multirow{2}{*}{ NPV } & \multirow{2}{*}{ Diagnostic accurac } \\
\hline & Positive & Negative & Total & & & & & \\
\hline Positive & 194 & 0 & 194 & \multirow{3}{*}{$88.2 \%$} & \multirow{3}{*}{$100 \%$} & \multirow{3}{*}{$100 \%$} & \multirow{3}{*}{$92.5 \%$} & \multirow{3}{*}{$95.2 \%$} \\
\hline Negative & 26 & 320 & 346 & & & & & \\
\hline Total & 220 & 320 & 540 & & & & & \\
\hline
\end{tabular}

TABLE 2: Comparison of frozen section diagnosis with final (paraffin) section diagnosis for the detection of sentinel lymph node metastasis

PPV, positive predictive value; NPV, negative predictive value

Table 3 compares the sensitivity, specificity, PPV, NPV, and diagnostic accuracy of intraoperative FS with respect to histological type. The sensitivity of FS for IDC was $88.3 \%$, whereas it was $85.7 \%$ for ILC. Similarly, the diagnostic accuracy of intraoperative FS for IDC and ILC was $94.9 \%$ and $97.2 \%$, respectively.

\begin{tabular}{|c|c|c|c|c|c|c|c|c|c|}
\hline \multirow{2}{*}{$\begin{array}{l}\text { Histological } \\
\text { type }\end{array}$} & \multirow{2}{*}{$\begin{array}{l}\text { Frozen section } \\
\text { diagnosis }\end{array}$} & \multicolumn{3}{|c|}{$\begin{array}{l}\text { Final (paraffin) section } \\
\text { diagnosis }\end{array}$} & \multirow{2}{*}{ Sensitivity } & \multirow{2}{*}{ Specificity } & \multirow{2}{*}{ PPV } & \multirow{2}{*}{ NPV } & \multirow{2}{*}{$\begin{array}{l}\text { Diagnostic } \\
\text { accuracy }\end{array}$} \\
\hline & & Positive & Negative & Total & & & & & \\
\hline \multirow{3}{*}{ IDC $(n=468)$} & Positive & 182 & 0 & 182 & \multirow{3}{*}{$88.3 \%$} & \multirow{3}{*}{$100 \%$} & \multirow{3}{*}{$100 \%$} & \multirow{3}{*}{$91.6 \%$} & \multirow{3}{*}{$94.9 \%$} \\
\hline & Negative & 24 & 262 & 286 & & & & & \\
\hline & Total & 206 & 262 & 468 & & & & & \\
\hline \multirow{3}{*}{ ILC (n = 72) } & Positive & 12 & 0 & 12 & \multirow{3}{*}{$85.7 \%$} & \multirow{3}{*}{$100 \%$} & \multirow{3}{*}{$100 \%$} & \multirow{3}{*}{$96.7 \%$} & \multirow{3}{*}{$97.2 \%$} \\
\hline & Negative & 2 & 58 & 60 & & & & & \\
\hline & Total & 14 & 58 & 72 & & & & & \\
\hline
\end{tabular}

TABLE 3: Comparison of frozen section diagnosis with final (paraffin) section diagnosis for the detection of sentinel lymph node metastasis with respect to histological type

IDC, infiltrating ductal carcinoma; ILC, infiltrating lobular carcinoma; PPV, positive predictive value; NPV, negative predictive value

Table 4 compares the sensitivity, specificity, PPV, NPV, and diagnostic accuracy of intraoperative FS with respect to tumor grade. The sensitivity of FS for grade G1, G2, and G3 tumors was $78.3 \%, 91.5 \%$, and $90.2 \%$, respectively. Similarly, the diagnostic accuracy of intraoperative FS for grade G1 tumors was $90 \%$ while it was $96.7 \%$ and $96 \%$ for grade $\mathrm{G} 2$ and G3 tumors, respectively. 


\section{Cureus}

\begin{tabular}{|c|c|c|c|c|c|c|c|c|c|}
\hline \multirow{2}{*}{ Tumor grade } & \multirow{2}{*}{$\begin{array}{l}\text { Frozen section } \\
\text { diagnosis }\end{array}$} & \multicolumn{3}{|c|}{$\begin{array}{l}\text { Final (paraffin) section } \\
\text { diagnosis }\end{array}$} & \multirow{2}{*}{ Sensitivity } & \multirow{2}{*}{ Specificity } & \multirow{2}{*}{ PPV } & \multirow{2}{*}{ NPV } & \multirow{2}{*}{$\begin{array}{l}\text { Diagnostic } \\
\text { accuracy }\end{array}$} \\
\hline & & Positive & Negative & Total & & & & & \\
\hline \multirow{3}{*}{$\begin{array}{l}\text { Grade } 1(n= \\
100)\end{array}$} & Positive & 36 & 0 & 36 & \multirow{3}{*}{$78.3 \%$} & \multirow{3}{*}{$100 \%$} & \multirow{3}{*}{$100 \%$} & \multirow{3}{*}{$84.4 \%$} & \multirow{3}{*}{$90 \%$} \\
\hline & Negative & 10 & 54 & 64 & & & & & \\
\hline & Total & 46 & 54 & 100 & & & & & \\
\hline \multirow{3}{*}{$\begin{array}{l}\text { Grade } 2(n= \\
214)\end{array}$} & Positive & 75 & 0 & 75 & \multirow{3}{*}{$91.5 \%$} & \multirow{3}{*}{$100 \%$} & \multirow{3}{*}{$100 \%$} & \multirow{3}{*}{$95 \%$} & \multirow{3}{*}{$96.7 \%$} \\
\hline & Negative & 7 & 132 & 139 & & & & & \\
\hline & Total & 82 & 132 & 214 & & & & & \\
\hline \multirow{3}{*}{$\begin{array}{l}\text { Grade } 3(n= \\
226)\end{array}$} & Positive & 83 & 0 & 83 & \multirow{3}{*}{$90.2 \%$} & \multirow{3}{*}{$100 \%$} & \multirow{3}{*}{$100 \%$} & \multirow{3}{*}{$93.7 \%$} & \multirow{3}{*}{$96 \%$} \\
\hline & Negative & 9 & 134 & 143 & & & & & \\
\hline & Total & 92 & 134 & 226 & & & & & \\
\hline
\end{tabular}

TABLE 4: Comparison of frozen section diagnosis with final (paraffin) section diagnosis for the detection of sentinel lymph node metastasis with respect to tumor grade

PPV, positive predictive value; NPV, negative predictive value

We compared the false-negative rate of FS with the final (paraffin) section diagnosis with respect to age, tumor grade, histological type, tumor size, and number of SLNs. The false-negative rate for grade G1 tumors was $21.7 \%$, which was higher than G2 and G3 tumors ( $8.5 \%$ and $9.8 \%$, respectively). Similarly, the falsenegative rate for cases where the number of SLNs was more than three was only $5.4 \%$, which was lower than cases with a single and two to three SLNs sent on FS (23.1 and 14.7\%, respectively). Similarly, the falsenegative rate of intraoperative FS for tumors measuring more than $5 \mathrm{~cm}$ was $0 \%$ as compared to $4.5 \%$ and $14.5 \%$ for tumors measuring less than or equal to $2 \mathrm{~cm}$, and tumors measuring between $2.1 \mathrm{~cm}$ and $5 \mathrm{~cm}$, respectively (Table 5). 


\section{Cureus}

Clinicopathological characteristics

Values

False-negative rate

Age groups

$\leq 50$ years, $\%$

$>50$ years, $\%$

Tumor grade

Grade 1, \%

Grade 2, \%

8.5

Grade 3, \%

Tumor size $(\mathrm{cm})$

$\leq 2 \mathrm{~cm}, \%$

$2.1-5 \mathrm{~cm}, \%$

$>5 \mathrm{~cm}, \%$

Histological type

IDC, \%

ILC, \%

Number of SLNs

$1, \%$

$2-3, \%$

$>3, \%$

5.4

TABLE 5: Comparison of the false-negative rate of the frozen section with the final (paraffin) section diagnosis with respect to clinicopathological characteristics

IDC, infiltrating ductal carcinoma; ILC, infiltrating lobular carcinoma; SLN, sentinel lymph node

\section{Discussion}

In this study, we noted a good overall diagnostic accuracy of FS for detecting breast cancer metastasis to axillary SLNs. The sensitivity of FS for detecting ILC metastasis to axillary SLNs was comparable to IDC (85.7\% vs. $88.3 \%$ for ILC and IDC, respectively). Alternatively, there was a substantial difference in the falsenegative rate of FS for detecting SLN metastasis with respect to tumor grade and the number of SLNs sent on FS intraoperatively.

Mukhtar et al. studied the false-negative rate of SLN biopsy for patients with ILC. They found no significant difference compared with other histological types; however, they concluded that an increasing number of SLN improves the diagnostic accuracy of SLN biopsy for detecting breast cancer metastasis [15]. Similar findings were noted in our study. Horvath et al. compared the diagnostic accuracy of intraoperative FS for 131 consecutive cases of ILC with 133 cases of IDC. They found no significant difference in terms of sensitivity, specificity, PPV, and NPV [16]. Concordant with these findings, in our study, the difference in the false-negative rate of FS for IDC and ILC was unsubstantial.

There is continuous debate and confusion among surgeons regarding the use of intraoperative FS for ILC of the breast. Guo R et al. in a study involving 707 cases of ILC reported a false-negative rate of $8.97 \%$. They emphasized that in their study, 183 of 196 cases with positive SLNs were clinically node-negative. Moreover, cases with false-negative FS were older with a fewer number of SLNs removed than patients with a falsenegative diagnosis [17]. These results further emphasize a need to evaluate more SLNs on FS than just a single lymph node.

The American College of Surgeons Oncology Group Z0011 (ACOSOG Z0011) randomized controlled trial 
evaluated the difference in overall survival of patients with SLN dissection (without axillary dissection) versus complete axillary clearance in patients undergoing breast conservation surgery. The trial enrolled patients with one or two positive SLNs. The median follow-up period was 9.3 years. The trial concluded that in patients with tumor stage $\mathrm{T} 1$ and $\mathrm{T} 2$ breast cancers undergoing breast conservation surgery, with clinically negative SLNs and histologically less than three positive SLNs should not undergo complete axillary clearance, as there was no significant difference in survival with or without axillary dissection in these patients [18]. In our series of patients, axillary dissection was not performed in patients with one or two positive SLNs on FS in patients undergoing breast conservation surgery. Alternatively, in patients undergoing mastectomy, axillary dissection was performed with even a single positive SLN macrometastasis on FS.

The limitations of our study include a fewer number of ILC cases than IDC and single-institution data. Therefore, we recommend large-scale prospective studies, including other histological subtypes of breast cancer evaluating the impact of other pathological and clinical factors impacting the sensitivity of intraoperative FS for detecting breast cancer metastasis to axillary SLNs.

\section{Conclusions}

Intraoperative FS is a useful technique to evaluate breast cancer metastasis to axillary SLNs. In our study, we noted that the difference in the diagnostic accuracy of FS for detecting IDC metastasis to axillary SLNs was unsubstantial as compared with ILC. However, the false-negative rate for low-grade tumors was higher than for high-grade tumors. Moreover, increasing the number of SLNs sent intraoperatively for FS improves the sensitivity for detecting breast cancer metastasis to axillary SLNs.

\section{Additional Information \\ Disclosures}

Human subjects: Consent was obtained or waived by all participants in this study. Animal subjects: All authors have confirmed that this study did not involve animal subjects or tissue. Conflicts of interest: In compliance with the ICMJE uniform disclosure form, all authors declare the following: Payment/services info: All authors have declared that no financial support was received from any organization for the submitted work. Financial relationships: All authors have declared that they have no financial relationships at present or within the previous three years with any organizations that might have an interest in the submitted work. Other relationships: All authors have declared that there are no other relationships or activities that could appear to have influenced the submitted work.

\section{References}

1. Hashmi AA, Edhi MM, Naqvi H, Faridi N, Khurshid A, Khan M: Clinicopathologic features of triple negative breast cancers: an experience from Pakistan. Diagn Pathol. 2014, 9:43. 10.1186/1746-1596-9-43

2. Hashmi AA, Aijaz S, Mahboob R, et al.: Clinicopathologic features of invasive metaplastic and micropapillary breast carcinoma: comparison with invasive ductal carcinoma of breast. BMC Res Notes. 2018, 11:531. 10.1186/s13104-018-3623-Z

3. Hashmi AA, Naz S, Hashmi SK, et al.: Prognostic significance of p16 \& p53 immunohistochemical expression in triple negative breast cancer. BMC Clin Pathol. 2018, 18:9. 10.1186/s12907-018-0077-0

4. Hashmi AA, Mahboob R, Khan SM, et al.: Clinical and prognostic profile of Her2neu positive (non-luminal) intrinsic breast cancer subtype: comparison with Her2neu positive luminal breast cancers. BMC Res Notes. 2018, 11:574. 10.1186/s13104-018-3677-y

5. Hashmi AA, Aijaz S, Khan SM, et al.: Prognostic parameters of luminal A and luminal B intrinsic breast cancer subtypes of Pakistani patients. World J Surg Oncol. 2018, 16:1. 10.1186/s12957-017-1299-9

6. Collier P, Hussain M, Popovic ZB, Griffin BP: Cardiac surveillance for anti-HER2 chemotherapy. Cleve Clin J Med. 2021, 88:110-6. 10.3949/ccjm.88a.19150

7. Hussain M, Hou Y, Watson C, et al.: Temporal trends of cardiac outcomes and impact on survival in patients with cancer. Am J Cardiol. 2020, 137:118-24. 10.1016/j.amjcard.2020.09.030

8. Hashmi AA, Faridi N, Khurshid A, et al.: Accuracy of frozen section analysis of sentinel lymph nodes for the detection of Asian breast cancer micrometastasis - experience from Pakistan. Asian Pac J Cancer Prev. 2013, 14:2657-62. 10.7314/apicp.2013.14.4.2657

9. Hashmi AA, Naz S, Ahmed O, et al.: Diagnostic accuracy of intraoperative touch imprint cytology for the diagnosis of axillary sentinel lymph node metastasis of breast cancer: comparison with intraoperative frozen section evaluation. Cureus. 2021, 13:e12960. 10.7759/cureus.12960

10. Hashmi AA, Edhi MM, Naqvi H, Khurshid A, Faridi N: Molecular subtypes of breast cancer in South Asian population by immunohistochemical profile and Her2neu gene amplification by FISH technique: association with other clinicopathologic parameters. Breast J. 2014, 20:578-85. 10.1111/tbj.12329

11. Hashmi AA, Munawar S, Rehman N, et al.: Invasive papillary carcinoma of the breast: clinicopathological features and hormone receptor profile. Cureus. 2021, 13:e13480. 10.7759/cureus.13480

12. Hashmi AA, Zia S, Yaqeen SR, et al.: Mucinous breast carcinoma: clinicopathological comparison with invasive ductal carcinoma. Cureus. 2021, 13:e13650. 10.7759/cureus.13650

13. Hashmi AA, Iftikhar SN, Munawar S, Shah A, Irfan M, Ali J: Encapsulated papillary carcinoma of breast: clinicopathological features and prognostic parameters. Cureus. 2020, 12:e11282. 10.7759/cureus.11282

14. Hashmi AA, Iftikhar SN, Haider R, Haider R, Irfan M, Ali J: Solid papillary carcinoma of breast: clinicopathologic comparison with conventional ductal carcinoma of breast. Cureus. 2020, 12:e11172. 


\section{Cureus}

10.7759/cureus.11172

15. Mukhtar RA, Brabham CE, Guo R, et al.: Accuracy of sentinel lymph node biopsy in invasive lobular carcinoma of the breast: Factors associated with false negatives. Breast J. 2021, 27:406-8. 10.1111/tbj.1416

16. Horvath JW, Barnett GE, Jimenez RE, Young DC, Povoski SP: Comparison of intraoperative frozen section analysis for sentinel lymph node biopsy during breast cancer surgery for invasive lobular carcinoma and invasive ductal carcinoma. World J Surg Oncol. 2009, 7:34. 10.1186/1477-7819-7-34

17. Guo R, Brabham CE, Fahrner-Scott K, et al.: Accuracy of sentinel lymph node biopsy in invasive lobular carcinoma of the breast. J Clin Oncol. 2020, 38:12604. 10.1200/JCO.2020.38.15_suppl.e12604

18. Giuliano AE, Ballman KV, McCall L, et al.: Effect of axillary dissection vs no axillary dissection on 10-year overall survival among women with invasive breast cancer and sentinel node metastasis. The ACOSOG Z0011 (Alliance) Randomized Clinical Trial. JAMA. 2017, 318:918-26. 10.1001/jama.2017.11470 